Bull. Austral. Math. Soc.

VOL. 57 (1998) [455-460]

\title{
ON A THEOREM OF BRYCE AND COSSEY
}

\section{A. Ballester-Bolinches and L.M. Ezquerro}

In this paper we characterise the subgroup-closed Fitting formations of finite groups which are saturated. This is an extension of the Bryce and Cossey result proving the saturation of all subgroup-closed Fitting formations of finite soluble groups.

\section{INTRODUCTION}

In 1972, Bryce and Cossey proved the following remarkable fact:

THEOREM. [1] A subgroup-closed Fitting formation of finite soluble groups is saturated.

As a consequence, the subgroup-closed saturated formations of finite soluble groups are precisely the primitive saturated formations.

Unfortunately, the above result is not true in the general universe $\mathfrak{E}$ of all finite groups as Doerk and Hawkes pointed out in [2, IX.1.6].

The natural question arising is to find necessary and sufficient conditions for a subgroup-closed Fitting formation to be saturated. The present paper is devoted to resolving this question.

Recall (see [2, Appendix $\beta]$ ) that given a group $G$ and a prime $p$ dividing $|G|$, there exists a group $E$, called the maximal $p$-Frattini extension of $G$, such that $E$ possesses a normal subgroup $A$ satisfying:

(a) $E / A$ is isomorphic to $G$ and $A \leqslant \Phi(E)$,

(b) $A$ is an elementary Abelian $p$-group,

(c) every other group extension of $G$ satisfying (a) and (b) is an epimorphic image of $E$.

The elementary Abelian normal $p$-subgroup $A$ can be regarded as a $\mathrm{GF}(p) G$ module. This module is called the $p$-Frattini module of $G$. Following the notation of [2], we write $A=A_{p}(G)$

We prove:

Received 23rd October, 1997

The first author is supported by Proyecto PB 94-0965 of DGICYT, Ministerio de Educación y Ciencia of Spain. The second author is supported by Proyecto PB 94-1048 of DGICYT, Ministerio de Educación y Ciencia of Spain.

Copyright Clearance Centre, Inc. Serial-fee code: 0004-9729/98 \$A2.00+0.00. 
THEOREM A. For a subgroup-closed Fitting formation $\mathfrak{F}$ the following are equivalent:

(i) If $G \in \mathfrak{F}$ is a primitive group of type 2 and $E_{p}$ is the maximal p-Frattini extension of $G$, then $E_{p} \in \mathcal{F}$, for every prime $p$ dividing $|\operatorname{Soc}(G)|$,

(ii) $\mathfrak{F}$ is saturated.

Our approach is not just an empty exercise in generalisation. In fact, Bryce and Cossey's proof depends heavily on the fact that if the Fitting subgroup $F(G)$ of a soluble group $G$ is a $p$-group, for some prime $p$, then $F(G) / \Phi(G)$ is a $G / \Phi(G)$-module over $\mathrm{GF}(p)$, the finite field of $p$ elements, such that $C_{G / \Phi(G)}(F(G) / \Phi(G))=F(G) / \Phi(G)$. This result is not true in the general finite universe. Therefore it is clear that we have to build a new proof of the result.

\section{STATEMENT OF PRELIMINARY RESULTS AND NOTATIONS}

All groups treated in this article will be finite. Most notation is standard and it is taken from [2]. The results which follow will be quoted in the proof of our main theorem. Some are given with reference but no proof. Others are so well-known as to require no proof here.

Recall that if $\mathfrak{X}$ is a class of groups, char $\mathfrak{X}$ denotes the set of all primes $p$ such that the cyclic group $C_{p}$ belongs to $\mathfrak{X}$, and $\sigma(\mathfrak{X})$ is the set of all primes $p$ such that $p$ divides the order of some group in $\mathfrak{X}$.

RESULT 2.1. If $\mathfrak{F}$ is a subgroup-closed Fitting formation, then char $\mathfrak{F}=\sigma(\mathfrak{F})$.

Result 2.2. [2, Appendix $\beta$ ] Let $G$ be a group and let $E$ be the maximal $p$-Frattini extension of $G$ for some prime $p$ dividing $|G|$. Denote by $A=A_{p}(G)$ the $p$-Frattini module of $G$. Then

$$
O_{p^{\prime} p}(G)=\bigcap\{\operatorname{Ker}(G \text { on } W): W \text { is a composition factor in } A\} .
$$

In other words, $O_{p^{\prime} p}(E / A)=\bigcap\left\{C_{E}(V / W) / A: V / W\right.$ is an $E$-chief factor below $\left.A\right\}$.

Proof: Denote $F_{G}(A)=\bigcap\{\operatorname{Ker}(G$ on $W): W$ is a composition factor in $A\}$. By block equivalence, the composition factors of $A$ belong to the first block and by a Theorem of Brauer (see [4, Theorem VII.14.8]) they are centralised by $O_{p^{\prime} p}(G)$. Therefore $O_{p^{\prime} p}(G) \leqslant F_{G}(A)$.

On the other hand, it is clear by definition that $F_{G}(A) \leqslant \operatorname{Ker}(G$ on $\operatorname{Soc}(A))$. By [3, Theorem 1] we know that $\operatorname{Ker}\left(G\right.$ on $\left.\operatorname{Soc}\left(A_{p}(G)\right)\right)=O_{p^{\prime} p}(G)$. Hence we obtain the equality $O_{p^{\prime} p}(G)=F_{G}(A)$.

In the sequel we suppose that $\mathfrak{F}$ is a subgroup-closed Fitting formation, $p$ is a fixed prime in char $\mathfrak{F}$ and the group $X$ is in $\mathfrak{F}$. 
After reading the proof of the above theorem of Bryce and Cossey, the following result is worth highlighting for later use. It holds in the general finite universe.

REsult 2.3.

(a) If $M, N$ are $\mathrm{GF}(p) X$-modules such that $[M] X \in \mathfrak{F}$ and $[N] X \in \mathfrak{F}$, then $[M \oplus N] X \in \mathfrak{F}$ and $[M \otimes N] X \in \mathfrak{F}$.

(b) Let $M$ be a $\operatorname{GF}(p) X$-module such that there exists a submodule $M_{0}$ of $M$ such that $[M, X] \leqslant M_{0}$ and $\left[M_{0}\right] X \in \mathfrak{F}$. Then $[M] X \in \mathfrak{F}$.

REsult 2.4. Let $M$ be a faithful $X$-module over $G F(p)$ such that $[M] X \in \mathfrak{F}$. Then $[V] X \in \mathfrak{F}$ for any $\operatorname{GF}(p) X$-module $V$.

Proof: For each natural number $r$, denote by $M^{(r)}$ the tensor power of $M$, $M^{(r)}=M_{1} \otimes \cdots \otimes M_{r}$, where $M_{i}$ is isomorphic to $M$ for each $i=1, \ldots, r$, regarded as GF $(p) X$-module according to the diagonal action. By Result 2.3 , it follows that $\left[M^{(r)}\right] X \in \mathfrak{F}$ for any $r$. In the proof of Steinberg's Theorem presented in [2, B,10.13], we can see that the regular $\operatorname{GF}(p) X$-module $R$ is isomorphic to a submodule of a direct sum of some tensor powers of $M$, since $M$ is faithful for $X$. Denote by $R_{0}$ such a direct sum. Then the semidirect product $G=\left[R_{0}\right] X$ is in $\mathfrak{F}$ by Result 2.3. Express $G$ as a product of its subgroups $R_{0}$ and $H=[R] X$ and $G=R_{0} H=F(G) H$. Since the formation $\mathfrak{F}$ is subgroup-closed, we have $H=[R] X \in \mathfrak{F}$. Let $V$ be any $X$-module and let $P(V)$ be its projective cover. Since $P(V)$ is a direct summand of a direct sum of copies of $R$, we have that $[P(V)] X \in \mathfrak{F}$. Finally, since $[V] X$ is a quotient group of $[P(V)] X$, we conclude that $[V] X \in \mathfrak{F}$.

Result 2.5. Assume that $[V] X \in \mathfrak{F}$ for every irreducible $\operatorname{GF}(p) X$-module $V$. Then $[W] X \in \mathfrak{F}$ for every $\mathrm{GF}(p) X$-module $W$.

Proof: Let $\mathfrak{M}$ be the class of all $\mathrm{GF}(p) X$-modules $V$ such that $[V] X \in \mathfrak{F}$. Then the class $\operatorname{Irr}_{\mathrm{GF}(p)}(X)$ of all irreducible $\mathrm{GF}(p) X$-modules is contained in $\mathfrak{M}$. Therefore

$$
K=\bigcap_{V \in \mathfrak{M}} \operatorname{Ker}(X \text { on } V) \leqslant \bigcap_{W \in \operatorname{Irr}_{\mathrm{GF}(p)}(X)} \operatorname{Ker}(X \text { on } W)=O_{p}(X) .
$$

Arguing as in the proof of the Bryce and Cossey theorem we have that $K=1$. Since $X$ is finite, there exists a finite number of $X$-modules in $\mathfrak{M}, V_{1}, \ldots, V_{n}$ say, such that $K=\bigcap_{i=1}^{n} \operatorname{Ker}\left(X\right.$ on $\left.V_{i}\right)$. Therefore $M=\bigoplus_{i=1}^{n} V_{i}$ is a faithful $X$-module over $\operatorname{GF}(p)$ such that $[M] X \in \mathfrak{F}$ by Result 2.3. Now the result follows by virtue of Result 2.4. REsult 2.6. Consider the class

$$
\mathfrak{F}_{p}=\left(G / C_{G}(H / K): G \in \mathfrak{F}, H / K \text { an Abelian } p \text {-chief factor of } G\right) .
$$


If $X \in \mathrm{R}_{0} \mathfrak{F}_{p}$, then $[V] X \in \mathfrak{F}$ for every $X$-module $V$ over $\operatorname{GF}(p)$.

Proof: Since $X \in \mathrm{R}_{0} \mathfrak{F}_{p}$, there exist normal subgroups $X_{1}, \ldots, X_{r}$ of $X$ such that $\bigcap_{i=1}^{r} X_{i}=1$ and $X / X_{i} \in \mathfrak{F}_{p}$. Therefore for each $i=1, \ldots, r$, there exists a group $G_{i} \in \mathfrak{F}$ and an Abelian $p$-chief factor $H_{i} / K_{i}$ of $G_{i}$ such that $X / X_{i} \cong G_{i} / C_{G_{i}}\left(H_{i} / K_{i}\right)$. Notice that $W_{i}=H_{i} / K_{i}$ is an $X$-module over $\operatorname{GF}(p)$ such that $\operatorname{Ker}\left(X\right.$ on $\left.W_{i}\right)=X_{i}$ for $i=1, \ldots, r$. Therefore $W=W_{1} \oplus \ldots \oplus W_{r}$ is a faithful $X$-module. Next we see that $[W] X \in \mathfrak{F}$. Let $i \in\{1, \ldots, r\}$. By $[2, I V, 1.5]$ the semidirect product $\left[W_{i}\right]\left(G_{i} / C_{G_{i}}\left(W_{i}\right)\right)$ belongs to $\mathfrak{F}$. Denote $W^{i}=\bigoplus_{j=1, j \neq i}^{r} W_{j}$ and $X^{i}=\left[W^{i}\right] X_{i}$. Now the subgroups $X^{i}$ are normal in $[W] X$. Moreover $([W] X) / X^{i}$ is isomorphic to $\left[W_{i}\right]\left(X / X_{i}\right) \in \mathfrak{F}$. Hence $[W] X \in \mathrm{R}_{0} \mathfrak{F}=\mathfrak{F}$ because $\bigcap_{i=1}^{r} X^{i}=1$. We apply Result 2.4 to deduce that $[V] X \in \mathfrak{F}$, for each $\mathrm{GF}(p) X$-module $V$.

In the sequel we consider a subgroup-closed Fitting formation $\mathfrak{F}$ satisfying the following property:

$(\alpha)$ If $G \in \mathfrak{F}$ is a primitive group of type 2 and $E_{p}$ is the maximal $p$-Frattini extension of $G$, then $E_{p} \in \mathfrak{F}$, for every prime $p$ dividing $|\operatorname{Soc}(G)|$.

RESULT 2.7. Let $G$ be a group in $\mathfrak{F}$ and let $H / K$ be a non-Abelian chief factor of $G$. If the prime $p$ divides the order of $H / K$, there exists a group $E \in \mathfrak{F}$ and an Abelian $p$-chief factor $A / B$ of $E$ such that

$$
G / C_{G}(H / K) \cong E / C_{E}(A / B) .
$$

ProOF: The group $\bar{G}=G / C_{G}(H / K)$ is a primitive group of type 2 in $\mathfrak{F}$ and $O_{p^{\prime} p}(\bar{G})=1$. Let $E$ be the maximal $p$-Frattini extension of $\bar{G}$ (notice that $p$ divides $|\bar{G}|$ ) and let $N$ be the elementary Abelian normal $p$-subgroup of $E$ such that $E / N \cong \bar{G}$ and $N \leqslant \Phi(E)$. Since $\mathfrak{F}$ satisfies the condition $(\alpha)$, the group $E$ is in $\mathfrak{F}$.

Obviously $N$ is contained in $C_{E}(A / B)$ for each $E$-chief factor $A / B$ below $N$. Suppose that $N$ is a proper subgroup of $C_{E}(A / B)$ for each $E$-chief factor $A / B$ below $N$. Then $S / N=S o c(E / N) \leqslant C_{E / N}(A / B)$ for every $E$-chief factor $A / B$ below $N$. This implies that

$$
S / N \leqslant \bigcap\left\{C_{E}(A / B) / N: A / B \text { is an } E \text {-chief factor below } N\right\} \cong O_{p^{\prime} p}(E / N)=1 \text {, }
$$

by Result 2.2. This is a contradiction. Consequently, there exists an $E$-chief factor $A / B$ below $N$ (and then $A / B$ is Abelian) such that $N=C_{E}(A / B)$. So $G / C_{G}(H / K)$ is isomorphic to $E / C_{E}(A / B)$ and the result is proved. 
RESULT 2.8. Consider the class

$$
\mathfrak{X}_{p}=\left(G / C_{G}(H / K): G \in \mathfrak{F}, H / K \text { is a } G \text {-chief factor, } p \in \pi(H / K)\right) \text {. }
$$

If $X \in \mathrm{R}_{0} \mathfrak{X}_{p}$, then $[V] X \in \mathfrak{F}$ for every $X$-module $V$ over $\operatorname{GF}(p)$.

Proof: Since $X \in \mathrm{R}_{0} \mathfrak{X}_{p}$, there exist normal subgroups $X_{1}, \ldots, X_{r}$ of $X$ such that $\bigcap_{i=1}^{r} X_{i}=1$ and $X / X_{i} \in \mathfrak{X}_{p}$, for each $i=1, \ldots, r$. Fix $i \in\{1, \ldots, r\}$. Then there exists a group $G_{i} \in \mathfrak{F}$ such that $X / X_{i}$ is isomorphic to $G_{i} / C_{G_{i}}\left(H_{i} / K_{i}\right)$, for some $G_{i}$-chief factor $H_{i} / K_{i}$. From Result 2.7, we can assume that $H_{i} / K_{i}$ is Abelian. This means that $X$ actually belongs to $R_{0} \mathfrak{F}_{p}$. Now the conclusion follows from Result 2.6 .

\section{Proof of Theorem A}

Let $\mathfrak{F}$ be a subgroup-closed Fitting formation. For each prime $p \in \operatorname{char} \mathfrak{F}$ we define

$$
f(p)=\mathrm{QR}_{0}\left(G / C_{G}(H / K): G \in \mathfrak{F}, H / K \text { is a } G \text {-chief factor, } p \in \pi(H / K)\right) .
$$

We show that $\mathfrak{F}=L F(f)$. This will imply that $\mathfrak{F}$ is saturated by a well-known theorem of Gaschütz (see [2, Theorem IV.4.6]).

It is clear that $\mathfrak{F} \subseteq L F(f)$. Suppose that $\mathfrak{F} \neq L F(f)$ and choose a group $G$ of minimal order in $L F(f) \backslash \mathfrak{F}$. Then $N=\operatorname{Soc}(G)$ is the unique minimal normal subgroup of $G$. If $N$ is non-Abelian, then $G / C_{G}(N) \in f(p)$ for each prime $p \in \pi(N)$, a contradiction. So $N$ is Abelian. Let $p$ be the unique prime dividing the order of $N$. Since $F(G)=O_{p}(G)=O_{p^{\prime} p}(G)$ and $O_{p^{\prime} p}(G)$ is the intersection of the centralisers of all $p$-chief factors, it follows that $G / F(G) \in f(p)$ and then $G / F(G) \cong X / T$ for some normal subgroup $T$ of a group $X \in \mathrm{R}_{0} \mathfrak{X}_{p}$. By Result 2.8 , it follows that $[W](G / F(G)) \in \mathfrak{F}$ for each $G / F(G)$-module $W$ over $\operatorname{GF}(p)$. So if $G$ is primitive, we have $N=F(G)$ and $G \cong[N](G / F(G)) \in \mathfrak{F}$, a contradiction. Therefore $N$ is contained in $\Phi(G)$. Let $H=G / N$ and let $V$ be an irreducible $H$-module over GF $(p)$. Since $N \leqslant \Phi(G)$, we have that $F(H)=F(G) / N$ and then $F(H)=O_{p}(H)$ is contained in $\operatorname{Ker}(H$ on $V)$. Therefore $V$ is a $G / F(G)$-module and $[V](H / F(H)) \in \mathfrak{F}$. The group $Z=[V] H$ has two normal subgroups $V$ and $F(H)$ such that $Z / V$ and $Z / F(H) \cong[V](H / F(H))$ are in $\mathfrak{F}$. Consequently $Z \in \mathrm{R}_{0} \mathfrak{F}=\mathfrak{F}$. By Result 2.5, $[M] H \in \mathfrak{F}$ for every $H$-module $M$ over $\operatorname{GF}(p)$. Therefore $Y=N \imath(G / N) \in \mathfrak{F}$. Since $G$ is isomorphic to a subgroup of $Y$ supplementing $F(Y)$, by [2, Theorem IV.1.14] it follows that $G \in \mathfrak{F}$. This is the final contradiction.

The converse follows easily. 


\section{REFERENCES}

[1] R.A. Bryce and J. Cossey, 'Fitting formations of finite soluble groups', Math. Z. 127 (1972), 217-223.

[2] K. Doerk and T. Hawkes, Finite soluble groups (Walter de Gruyter, Berlin, New York, 1992).

[3] R.L. Griess and P. Schmid, 'The Frattini module', Arch. Math. 30 (1978), 256-266.

[4] B. Huppert and N. Blackburn, Finite groups II (Springer-Verlag, Berlin, Heidelberg, New York, 1982).

Departament d'Àlgebra

Facultat de Ciencies Matemàtiques

Universitat de València

C/ Dr. Moliner $\mathbf{5 0}$

46100 Burjassot (València)

Spain
Departamento de Matemática e Informática

Universidad Pública de Navarra

Campus de Arrosadía

31006 Pamplona (Navarra)

Spain 